\title{
NUMERICAL SIMULATION OF SEPARATION OF CIRCULATING TUMOR CELLS FROM BLOOD STREAM IN DETERMINISTIC LATERAL DISPLACEMENT (DLD) MICROFLUIDIC CHANNEL
}

\author{
F. Khodaee \\ Biological Fluid Mechanics Research Laboratory \\ Biomechanics Department \\ Amirkabir University of Technology \\ Tehran, Iran
}

\author{
S. Movahed ${ }^{*}$ \\ Department of Mechanical Engineering \\ Amirkabir University of Technology \\ Tehran, Iran
}

\author{
N. Fatouraee \\ Biological Fluid Mechanics Research Laboratory \\ Biomechanics Department \\ Amirkabir University of Technology \\ Tehran, Iran
}

\section{F. Daneshmand}

Department of Bioresource Engineering

McGill University

Montreal, Canada

\begin{abstract}
Deterministic Lateral Displacement (DLD) microfluidic devices provide a reliable label-free separation method for detection of circulating tumor cells (CTCs) in blood samples based on their biophysical properties. In this paper, we proposed an effective design of the DLD microfluidic device for the CTC separation in the blood stream. A typical DLD array is designed and numerical simulations are performed to separate the CTC and leukocyte (white blood cells) in different fluid flow conditions. Fluid-Solid Interaction method is used to investigate the behaviour of these deformable cells in fluid flow. In this study, the effects of critical parameters affecting cell separation in the DLD microfluidic devices (e.g. flow condition, cell deformability, and stress) have been investigated. The obtained results show that unlike leukocytes, the CTC's motion is independent of the flow condition and is laterally displaced even in higher Reynolds number. Larger cells (CTCs) cannot intercept the low-velocity fluid near the wall of the posts; thus, they move faster and become separated from leukocytes. To reduce the cellular stress during separation process, which causes increase of cell viability and more effective design of microfluidic device, the results obtained here may be used as a significant design parameter for the DLD fabrication.
\end{abstract}

Keywords: Deterministic lateral displacement (DLD), Circulating tumor cell (CTC), Microfluidics, Fluid-solid interaction (FSI).

\section{INTRODUCTION}

Circulating tumor cells (CTCs) are a small number of cells in blood of cancerous patients that were first discovered in 1869 by Thomas Ashworth [1]. These cells are similar to the primary tumors and can be found in the blood stream. It is believed that these cells implement formation of the secondary tumors during metastasis [2]. Therefore, detection of these extremely rare cells within the blood stream may provide an important prospect for cancer prognosis. Different strategies have been proposed to capture, enumerate and characterize the CTCs [3,4]. A key limitation in capturing and analyzing the CTCs is their extreme rarity in the blood relative to the hematological cells, which imposes formidable technical and analytical challenges. Since the CTCs can be found as low as 1 in 109 erythrocytes and 1 in 107 leukocytes, the challenge in isolating the CTCs is effective depletion of these cell types [5]. The mature erythrocytes have distinct physical, chemical, and biological properties which allow them to be easily removed from the blood. However, the leukocytes share many properties with the CTCs, leading to high levels of leukocyte contamination in many sep-

\footnotetext{
* Corresponding author (smovahed@aut.ac.ir)
} 
aration methods. Thus, effective discrimination of the CTCs from the leukocytes is the key functional requirement of any separation technique. Different biochemical and physical separation methods have been proposed to separate the CTCs from the other blood components [6]. The biochemical CTC separation methods are typically based on a combination of affinity capture of cell surface antigens to enrich for potential target cells and fluorescent labelling of intracellular markers to identify the CTCs [3]. Existing biochemical separation processes discriminate the CTCs from the hematological cells using antigens expressed in epithelial cells. An established system developed using this approach has been marketed as the Veridex Cell Search System (Raritan, NJ, USA), and has received FDA approval for clinical enumeration of the CTCs. However, this technique is expensive, laborious and time-consuming; variable efficiencies of enumeration have also been reported for this method [7]. The physical CTC separation methods are based on differences in the physical properties of the CTCs including cell size, shape, deformability, density, electrical polarizability, and magnetic susceptibility compared to leukocytes. These methods are label-free, and therefore are able to avoid the epithelial antigen bias of existing biochemical methods.

By recent advancements in microfabrication techniques, the idea of using microfluidic devices for different biological and biomedical applications, such as CTC separation, has become increasingly common [8-11].The microfluidic devices offer many advantages such as significant reduction in the consumption of biological samples and reagents. Moreover, the continuous flow in the channels of the microfluidic devices can simulate the real situation in a living biological system. Many studies have suggested using microfluidic devices for the label-free separation of the CTCs from the other blood components based on their physical properties [12-16]. The biophysical and biomechanical properties of the CTCs are important because of their potential to be used to discriminate the CTCs from the leukocytes. In physical-based methods, size and deformability play important roles in the CTCs separation. Filtration of the cells through an array of micro-scale constrictions [17,18], inertia separation using inertial effects including drag forces from Dean flows, shear gradient lift forces, and wall-effect lift forces that can be balanced against each other to enable size based separation $[15,16]$ and dielectrophoresis (DEP) which is capable of discriminating between blood cells and tumor cells on the basis of the cell size $[19,20]$ are some typical methods which benefit the size and deformability difference between the CTCs and other blood components for their separation.

One prominent design in low Reynolds number regime is deterministic lateral displacement (DLD) which was first proposed by Huang and colleagues [21]. They exploit the hydrodynamic size differences between the nucleated and the non-nucleated cells. This is one of the mechanisms of particle separation that uses the laminar properties of the microfluidics in a periodic array of posts to sort the particles based on their size. In this method, unlike the cells larger than critical size, the cells smaller than the critical size will follow streamlines and pass through an array of the posts with no net lateral displacement, while the cells larger than the critical size will be shifted to the different streamline; when they are confronted with obstructions they are laterally displaced from the original streamline. This method has been widely used for the cell separation (e.g. separation of the blood components) [22-24], separation of living parasites from human blood [25,26], purification of fungal spores [27], bacterial chromosomes [28], separation of healthy and malignant lymphocytes, [29] and separation of chromosomal bacterial DNA of differing lengths [21].

Experimental studies have also been conducted to develop the DLD microfluidic devices for the CTC separation. As an example, Liu et al. [30] performed the experimental and theoretical studies and proposed the DLD microfluidic design for rapid and label-free cancer cell separation and enrichment from diluted peripheral whole blood; their proposed design is based on size-dependant hydrodynamic forces acting on the different cells [30]. Loutherback et al. [31] proposed the microfluidic design based on the DLD array to separate the CTCs from the blood stream. Their results show that more complex heterogeneous triangle arrays will allow the separation of a wide range of object sites with no clogging issue [31]. An integrated microfluidic lab-on-a-chip has also been introduced by Liu et al. [32] for effective and rapid isolation and capture of the CTCs from the blood.

Numerical studies were also conducted to investigate the DLD mechanism. As an example, Long et al. [33] numerically investigate array geometries with rational row-shift fractions in the DLD devices. They considered the effects of both advection and diffusion in their simulations; their results show that using needle-like posts decreases the shift in critical size due to the diffusion. Their results are valid for the devices where the post separation is on the same scale as the post diameter. Using numerical simulations, Quek et al. [34] studied the separation of deformable bodies, such as capsules, vesicles, and cells, in the DLD. Their results prove that in addition to the zigzag and laterally displaced trajectories that have been observed experimentally, there exists a third type of trajectory which is called dispersive. This type of movement (dispersive) is only observed for large and rigid particles whose diameters are approximately more than half the gap size between micro-pillars and the particles whose stiffness exceeds approximately 500MPa. Another numerical study performed by D'Avino [35] shows that the critical particle size in the DLD device can be tuned by using non-Newtonian fluids as suspending liquid. Many other numerical studies were conducted to examine particle motions in the DLD microfluidic devices [36,37].

So far, theoretical studies on the microfluidic based devices for separation of the CTCs from the blood components have lagged behind experimental ones, though they are completely essential to boost the current understanding of the mechanism of the separation, 
optimize the functionality of the current proposed microfluidic designs for the CTC separation, and reduce the side effects of the proposed system. To the best of our knowledge, there is no comprehensive numerical study on the CTC motions and separations in the DLD microfluidics devices; and all the previous researches focused on the experimental aspects of CTC separation by this method. To obtain the best results, deformability of the CTCs should be considered for the simulations. Also, most of the previous studies considered separation of the rigid particles in the DLD microfluidic devices; their results may not be extended to the deformable particles such as CTCs in the DLD microfluidic devices.

In this paper, a numerical study is performed to investigate the mechanism of the CTC separation by the DLD microfluidic device. In contrast to most of the previous numerical studies on DLD, here, the deformability of the particles is considered and a fluid-solid interaction algorithm is used to simulate their motion in the DLD arrays. Effect of different flow rates on the mechanism of the separation is investigated in our simulations. The rest of this article is organized as follows: In the next section, the governing equations and computational model of this study are provided; in section 3, the results of our simulations are presented. After that, the obtained results of this study are presented and are discussed. The concluding remarks are provided at the end of the article.

\section{MATERIAL AND METHODS}

\subsection{Theory}

As it was mentioned before, in the DLD microfluidic devices the particles will be separated by size. For each DLD design, the critical size of particles and the criteria of particle separation should be estimated carefully. Inglis et al. have developed a theory to determine the critical particle size in the DLD designs [36]. Based on their proposed theory, each gap can be divided by the stall lines into flow streams that have equal fluid flux. For each particle, if its radius is larger than the width of these stall lines, it will be forced to change its flow stream; otherwise, it will remain in the same stall line. Deterministic lateral displacement separation method is based on the asymmetric bifurcation of the laminar flow around an array of micro obstacles. These micro obstacles are shifted a specific distance $(\varepsilon \lambda)$ from the previous row (Fig. 1). This shifted distance $(\varepsilon \lambda)$, together with the gap size $(g)$ and the centerto-center distance between two adjacent obstacles $(\lambda)$, determines the critical separating particle size. When the particles with different sizes flow through these micro obstacles, they will follow specific pathways "deterministically". The particles smaller than the critical separating size will periodically change their path lines and follow a "zigzag" mode, whereas particles larger than the critical separating size will keep getting "bumped" when they travel to the next row of the obstacles and thus be displaced to one direction. If the flow profile is generated by the pressure gradient and it is parabolic, one can obtain the critical diameter $\left(D_{c}\right)$ by using the following equations:

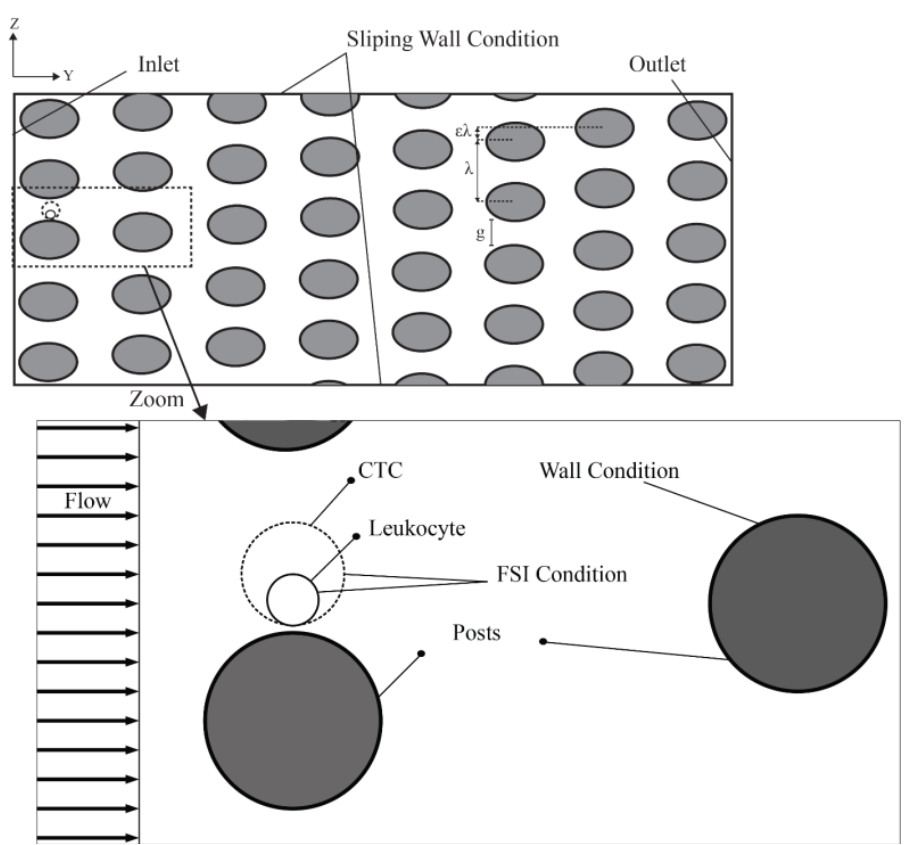

Fig. 1 Schematic diagram of numerical domain in this study. CTC and leukocyte are modeled here in a fluid field with flow inlet containing circular array posts.

$$
D_{c}=g\left[1+2 \delta+\frac{1}{2 \delta}\right]
$$

where $\delta$ is a parameter defined as shown below:

$$
\delta=\left[\frac{1}{8}-\frac{\varepsilon}{4}+\sqrt{\frac{\varepsilon}{16}(\varepsilon-1)}\right]^{1 / 3}\left(-\frac{1}{2}-i \frac{\sqrt{3}}{2}\right)
$$

In the above equation, $\varepsilon$ is the row shift fraction which is equal to $\varepsilon \lambda / \lambda, g$ is the gap distance and $i$ is the imaginary unit. The slope of post arrays is a function of row shift fraction $(\varepsilon)$ and is referred as tiled angles $(\tan (\varepsilon))$.

\subsection{Geometry and Material Properties}

Unlike hematological cells, the diameter of the CTCs is bigger than $15 \mu \mathrm{m}$ [37]; thus, the design of the DLD post array should be based on the critical separation size of $15 \mu \mathrm{m}$. Using the theory of critical separation size [36] and referring to previous research on this topic $[23,31,32]$, the parameters of Table 1 were chosen for the numerical simulations of this study.

In the DLD microfluidic devices, cell deformation has a decisive effect on the cell separation that may change the trajectories of particles passing through arrays. Comparison of motion of rigid and deformable particles shows that the deformable cells have obeyed different critical separation sizes [38,39]. Regarding these researches, the deformability of the cells should be considered in the simulations. The results of Atomic Force Microscopy (AFM) show that the elastic 
Table 1 Geometrical parameters of the DLD microfluidic device design for numerical simulations in this study.

\begin{tabular}{|c|c|c|c|}
\hline $\begin{array}{c}\text { Critical } \\
\text { diameter size } \\
(\mu \mathrm{m})\end{array}$ & $\begin{array}{c}\text { Gap size } \\
(\mu \mathrm{m})\end{array}$ & $\begin{array}{c}\text { Row shift } \\
\text { space }(\mu \mathrm{m})\end{array}$ & Tiled angle \\
\hline 15 & 50 & 70 & $3.2^{\circ}$ \\
\hline
\end{tabular}

module of the CTCs is in the range of $0.23 \sim 1.1 \mathrm{kPa}$ [40]. Also, leukocyte mechanical behaviour is modeled as Maxwell fluid [41] in which stress ( $\tau$ ) and strain $(\gamma)$ are related by:

$$
\dot{\gamma}=\frac{\tau}{2 \mu_{\text {cell }}}+\frac{\dot{\tau}}{2 G_{\text {cell }}}
$$

where a superimposed dot indicates time differentiation of the variable and $\mu_{\text {cell }}=31$ Pa.s and $G_{\text {cell }}=186 \mathrm{~Pa}$ are coefficients of viscosity and elastic shear modulus, respectively; a constant tension of $31 \mathrm{pN} / \mu \mathrm{m}$ is assumed around the cell to model behaviour of membrane [42].Before performing experiments, the blood samples were usually diluted ten times; thus, the fluid was assumed to be water with viscosity of $1 \mathrm{cP}$ and density of $1000 \mathrm{~kg} / \mathrm{m}^{3}$.

\subsection{Governing Equations}

In this study, as the cell and the fluid affect each other, the fluid-solid interaction (FSI) approach is used [43]. In the FSI solutions, the arbitrary LagrangianEulerian formulation (ALE) has been used. An ALE formulation includes both pure Eulerian and pure Lagrangian formulations where an arbitrary referential coordinate should be introduced in addition to the Lagrangian and Eulerian coordinates. The governing equations for the ALE formulation are the conservation of mass, the momentum equation, and the energy equation. Proper boundary conditions should be applied to consider fluid-structure interaction at the surface of cell. These governing equations and boundary conditions are summarized as follow:

The conservation of mass equation:

$$
\frac{\partial \rho}{\partial t}=-\rho \frac{\partial v}{\partial x_{i}}-w_{i} \frac{\partial \rho}{\partial x_{i}}
$$

The momentum equation:

$$
\frac{\partial v}{\partial t}=-\left(\tau_{i j, j}+\rho \mathrm{b}_{i}\right)-\rho w_{i} \frac{\partial v_{i}}{\partial x_{j}}
$$

Where the stress tensor is described as follows:

$$
\tau_{i j}=-p \delta_{i j}+\mu\left(v_{i, j}+v_{j, i}\right)
$$

The energy equation:

$$
\frac{\partial E}{\partial t}=-\left(\tau_{i j} v_{i, j}+\rho b_{i} v_{j}\right)-\rho w_{j} \frac{\partial E}{\partial x_{j}}
$$

where, in recent equations $\rho$ is density, $b$ is the body force, $E$ represents the internal energy and $v$ and $w$ are the material and reference velocities, respectively.

The fundamental conditions applied to the fluidstructure interfaces are the kinematic condition (or displacement compatibility):

$$
\underline{d}_{f}=\underline{d}_{s}
$$

and the dynamic condition (or traction equilibrium):

$$
n \cdot \underline{\tau}_{f}=n \cdot \underline{\tau}_{s}
$$

where $\underline{d}_{f}$ and $\underline{d}_{\text {s }}$ are the fluid and solid displacements, respectively; $\underline{\tau}_{f}$ and $\underline{\tau}_{s}$ are the fluid and solid stresses, in that order. The underlining denotes that the values are defined on the fluid-structure interfaces only.

\subsection{Numerical Simulation}

The coupled fluid and structure model was solved by the finite element package ADINA (Adina R \& D Inc., Watertown MA, USA). We have implemented the steered adaptive mesh repair procedure, coarsening meshes in the simulation of the fluid flow [44]. To discretize the fluid domain, the freeform meshing with 3-node triangular elements in 2D flows was employed. Quadrilateral 2-D Solid type elements having 9 nodes were used for the solid domain. The direct computational two-way coupling FSI method is employed, as this method is faster than the iterative. In this method, the fluid and the solid equations are combined and treated in a unit system. The Newton-Raphson iterative method was used to solve the discretized equations for both the solid and the fluid. Here, 500 iterations per step were used for the cells and blood models in the Newton-Raphson scheme. Initially, the particle was fixed by a spring to reach the steady state condition in fluid domain and with beginning the steady state condition, the spring element was removed by element death as it was implemented by Vahidi et al. [45]. No-slip and FSI boundary conditions are assumed on the wall of arrays and the wall of the CTC and the leukocyte, respectively. Also, a uniform constant fluid velocity at the inlet is imposed as boundary condition and a free traction at outlet (Fig. 1).

To improve the accuracy of numerical simulation, mesh independency is investigated using the magnitude of fluid velocity between two posts. Increasing the number of elements more than 9000 did not affect the results; so, this number of elements assures the accuracy of our simulation and was used for simulations (Fig. 2). An exemplary mesh of fluid and solid domains is shown in Fig. 3. 


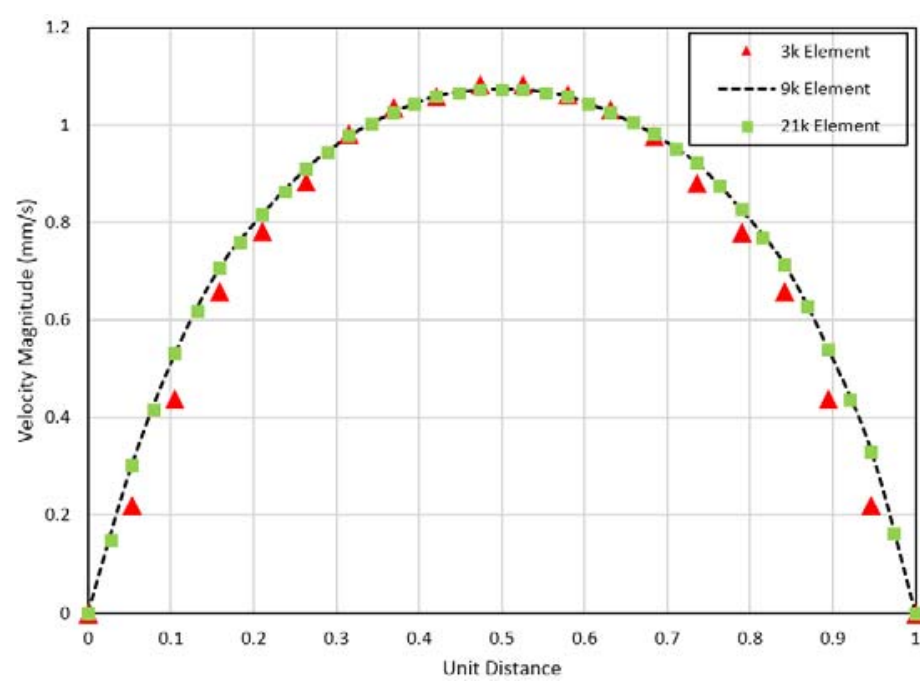

Fig. 2 To analyze mesh independency the velocity magnitude between two posts is characterized for different number of elements. Increasing the number of elements to more than 9,000 does not change the velocity profile so discretization with 9,000 elements was implemented in this study.

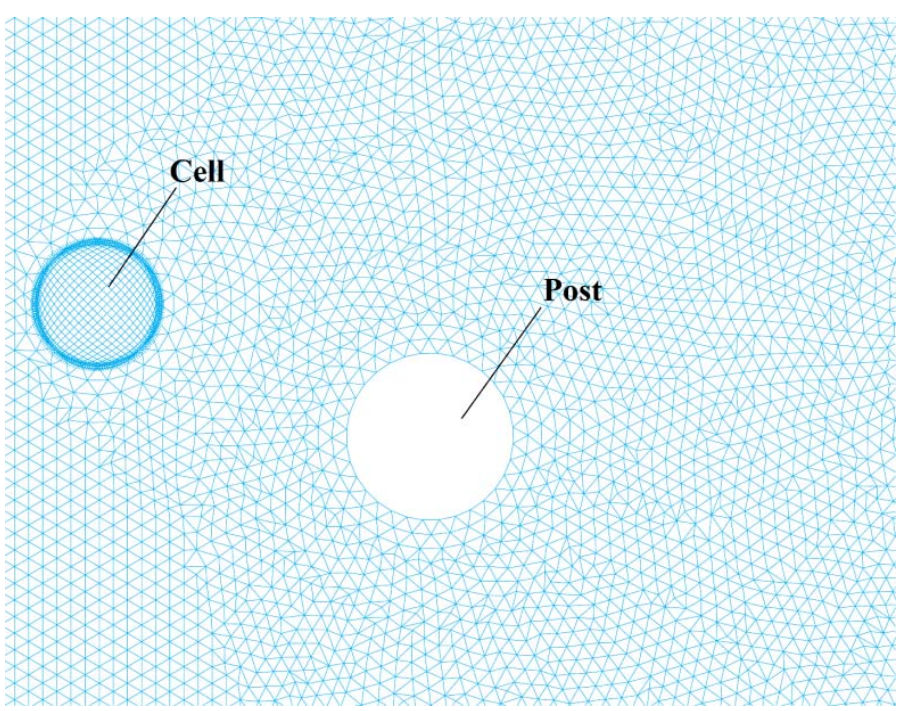

Fig. 3 Typical Discretized numerical domain of fluid and structure parts.

\section{RESULTS AND DISCUSSION}

As it was mentioned previously, in this study, the motion and separation of the CTC and the leukocyte cells are investigated in the DLD microfluidic devices. Different flow regimes were assumed to examine the effectiveness of the assumed DLD microfluidic device for the CTC separation. Here, we simulated the cells in two different Reynolds numbers (0.1 and 1); results including displacement, velocity, acceleration, deformation and stress of cells will be presented.

\subsection{Cell Motion}

Large particles cannot intercept the low-velocity fluid near the wall of the posts; thus, the bigger cells
(CTCs) move faster and become separated from leukocytes. Motion of the cells in the microfluidic DLD devices is described by their trajectories through array posts. Depending on the size of the cells and the Reynolds number, the cells will follow different trajectories. Thus, these parameters should be defined precisely to enhance the performance of the device. Figure 4 shows displacement of the cells under different flow conditions. Two different flow conditions that are represented by Reynolds number around the circular posts are assumed in the simulations. In computing the Reynolds number, the characteristic diameter of circular posts and the velocity magnitude of the inlet fluid are considered. This graph demonstrates trajectory of the cells. The cells are all initially released from the same point which is assumed as origin of coordinate system (see Fig. 4). This figure shows the trajectory of the two cell types (e.g. CTC and white blood cell); the locations of the circular array posts of the DLD design are depicted by multiple arrows on each graph.

As it is shown in Fig. 4, both CTC and white blood cell (hereafter WBC) initially follow the same path; as they go forward, the effect of posts causes them to pass through different trajectories. For the higher Reynolds number ( $\mathrm{Re}=1$, gray lines in Fig. 4), the cells are laterally displaced and move farther from the posts. When the Reynolds decreases to 0.1 , the cells encounter more profound wall effect due to their motion close to the posts; for WBC which is smaller, a zigzag motion will be triggered through posts (black dotted line in Fig. 4). In this condition, the possibility of contact between the cells and the posts increases. As an example, for $\operatorname{Re}=0.1$, the CTC contact the $4^{\text {th }}$ post which causes a jumped vertical motion of cell near the post.

Figure 5 shows the trajectory of the WBCs under different flow conditions; colours represent velocity magnitude of flow field. The velocity field in both conditions follows a similar pattern. Laminar flow is observable and a parabolic profile of velocity can be detected between posts. Maximum velocity magnitude occurs in the middle of vertical gaps. Therefore, when the cells approach this area, they are influenced by a momentum forcing them downward. As it was previously mentioned, the WBCs in the lower Reynolds number follow the zigzag motion through posts which is clearly visible in Fig. 5.

Motion of the CTC is also shown in Fig. 6. The CTC moves similar to the WBC, however, because of their bigger size, their interaction with the flow field is more considerable. As it is shown in Fig. 6, the velocity field changes due to the motion of the CTC; more specifically, the alteration of flow field due to the CTC motion is observable for the lower Reynolds number. In spite of the WBC, CTC motion is not affected by the flow condition; for both values of the Reynolds number ( $\operatorname{Re}=1$ and $\operatorname{Re}=0.1$ ), it moves laterally. The variation and pattern of velocity during the cell's passage through posts is depicted in Fig. 7. Generally, increase of the Reynolds number intensifies the velocity of cells. As it is shown in Fig. 7, for the higher Reynolds number, the cells move faster. The maximum 


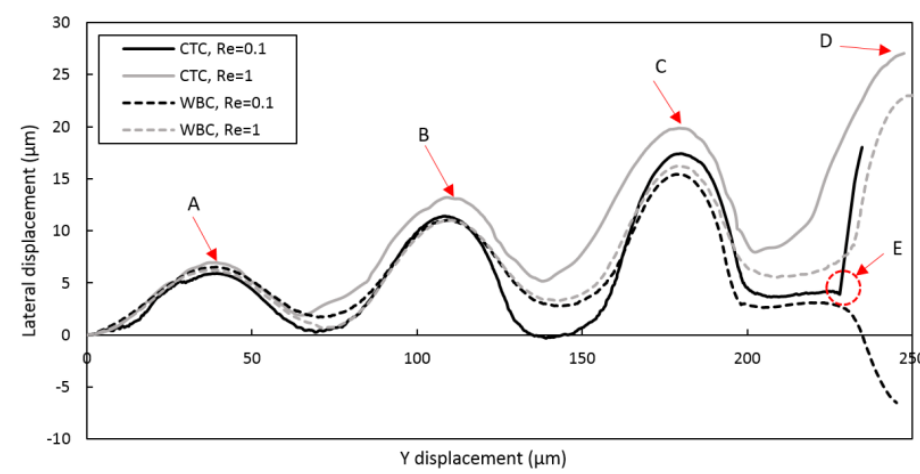

Fig. 4 Trajectory of different cells, e.g. CTC and white blood cell (WBC) with different flow conditions through 2D array, here A, B, C and $\mathrm{D}$ represent location of first, second, third and fourth posts respectively. E shows the situation whereby the cell is near to contacting with a post.

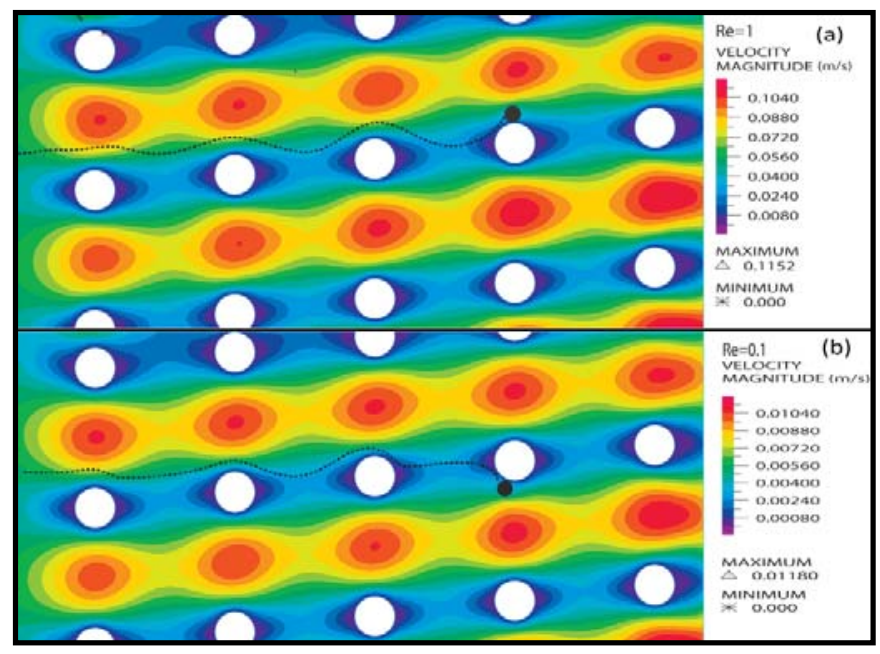

Fig. 5 Leukocytes trace through array with velocity field in (a) $\operatorname{Re}=1$ and (b) $\operatorname{Re}=0.1$. The colour bars do not have the same range.

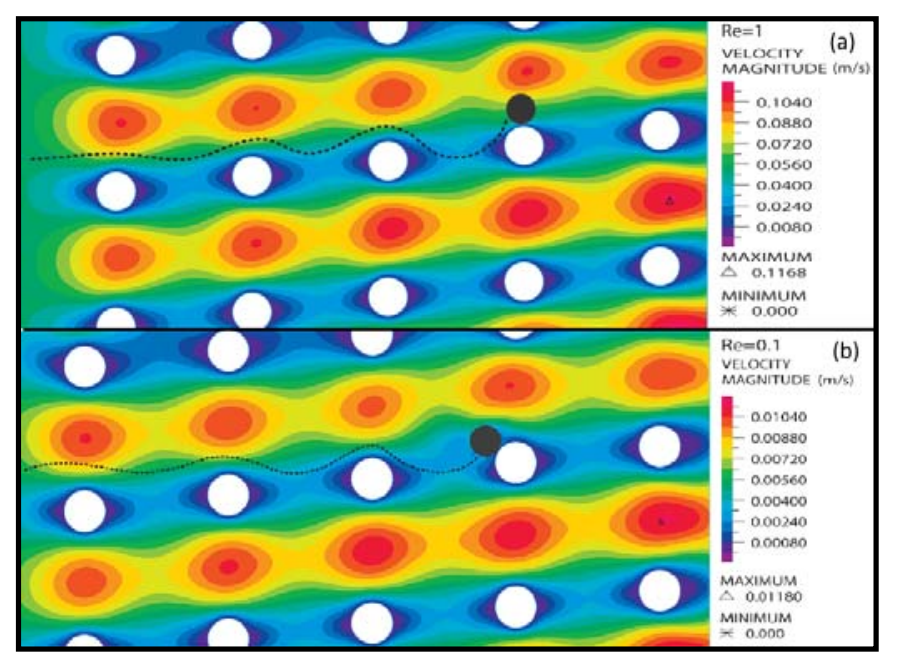

Fig. 6 CTC trace through array with velocity field in (a) $\mathrm{Re}=1$ and (b) $\mathrm{Re}=0.1$. The colour bars do not have the same range.

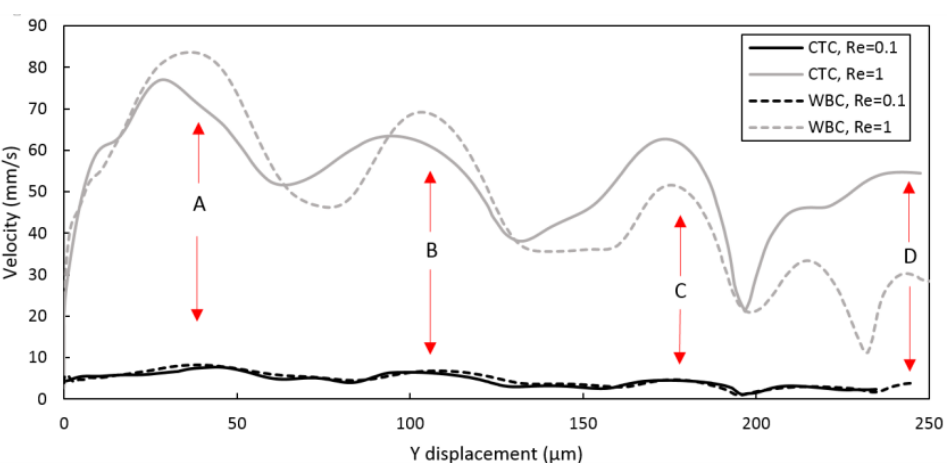

Fig. 7 Illustration of velocity magnitude of cells versus longitudinal displacement through array. Location of posts is indicated in the graph, here $\mathrm{A}, \mathrm{B}, \mathrm{C}$ and $\mathrm{D}$ represent location of first, second, third and fourth posts respectively.

speed of the cell occurs when the cells move above posts; immediately after that, speed of the cells decreases. No significant difference is observed between the CTCs and the WBCs in their velocity, however, the WBCs experience more oscillation in their speed. These results are similar to previous researches on the cell trajectory and fluid field of the DLD microfluidic device $[34,35,46]$.

Vertical component of the velocity ( $z$ velocity) provides more information about the cells motion through barriers and their tendency to move laterally. When the has a tendency to move downward, $z$ component of velocity continues to remain negative; as an example, the black dotted line of Fig. 8 shows the $z$-component of velocity of WBC $(\operatorname{Re}=0.1)$.

Vectors of velocity in the fluid field for a typical case is shown in Figs. 9(a) and 9(b). It shows the magnitude and direction of the velocity vectors around posts and the cell; it also indicates that the flow guides the cell though the barriers. As an example, in Fig. 9(a), the cell decelerated and its velocity is less than the velocity of surrounding fluid where it is completely vice versa for the case of Fig. 9(b).

The behavior and movement of the particles are mainly affected by drag force at low Reynolds numbers (laminar flows). When the stress acting on the cells due to the drag force (the dominant force induced to the cell on laminar flow) dramatically increases, the cells have more tendencies to change their direction through the posts which causes a zigzag motion. For the bigger cells (CTCs), the velocity gradient acting on them is stronger and the zigzag motion is more tangible (see Fig. 10). As it is shown in Fig. 9, the presence of posts makes the velocity field very complex and causes more velocity gradient (this gradient is higher near the posts and can be sensed more by the cells moving there). Therefore, for the larger cells the velocity gradient has a major role in the separation procedure.

\subsection{Cell Stress and Deformation}

Unlike previous research on the DLD [34,35], the current study aims to present a prospective of the cells' stress and deformation and their effects on the cell motion. Equation of effective stress is shown by: 


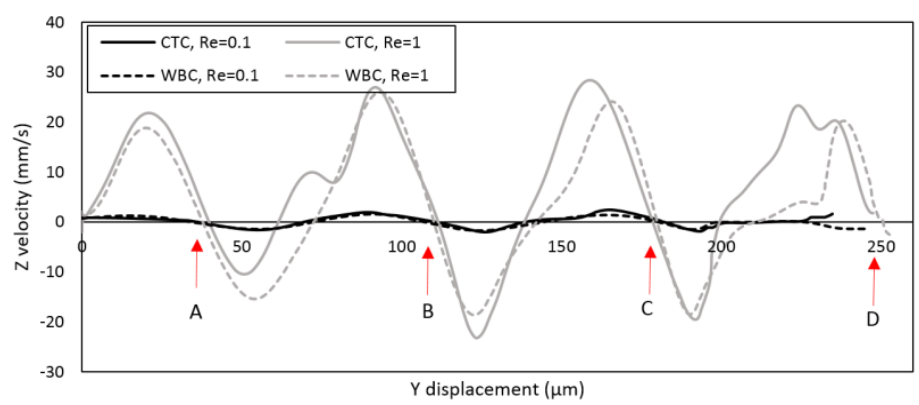

Fig. $8 \quad z$-component of velocity versus longitudinal displacement during cells motion through array. Location of posts is indicated in the graph, here $\mathrm{A}, \mathrm{B}, \mathrm{C}$ and $\mathrm{D}$ represent location of first, second, third and fourth posts respectively.

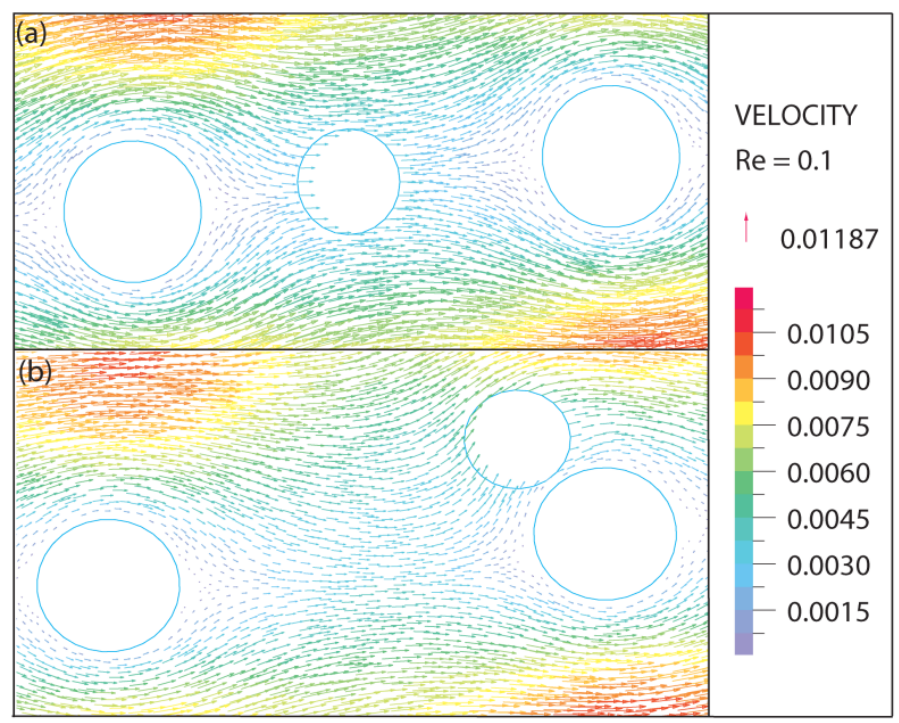

Fig. 9 Velocity vectors around particle moving (a) between two posts and (b) beside a post.

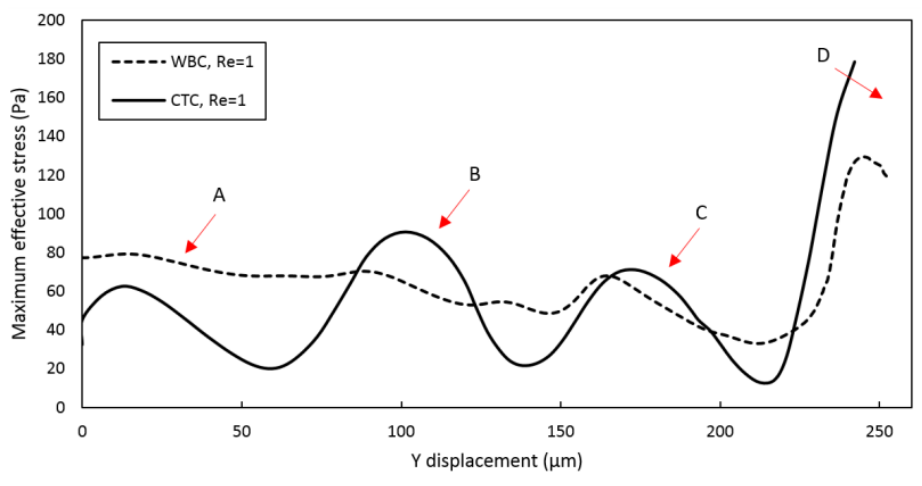

Fig. 10 Maximum amount of effective stress acting on cells versus longitudinal displacement during their motion through array. Location of different posts is indicated in the graph, here $A$, $\mathrm{B}, \mathrm{C}$ and $\mathrm{D}$ represent location of first, second, third and fourth posts respectively.

$$
\bar{\sigma}=\sqrt{\frac{3}{2} S_{i j} S_{i j}}
$$

where $S_{i j}$ are the deviatoric stresses

$$
S_{i j}=\sigma_{i j}-\frac{1}{3} \delta_{i j} \sigma_{k k}
$$

and $\delta_{i j}$ is the Kronecker Delta and $\sigma_{i j}$ is the stress.

As the cells move forward, for the case of $\mathrm{Re}=1$, Fig. 10 shows maximum amount of effective stress on the cell. As it is shown in this Figure, the peak of maximum effective stress occurs before reaching the zenith of each post. Generally, the WBC experiences less stress than the CTC and it might be because of their smaller size and different mechanical properties. Maximum effective stress increases by increasing the chance of contact between the cells and the posts.

As it can be seen in Fig. 10, the maximum amount of cell's stress occurs when the cell reaches the post. The contour of effective stress acting on the leukocyte and the CTC approaching the posts is depicted in Fig. 11. Also, the maximum deformation occurs when the cells move beside the posts; greater proximity of the cells to posts causes more stress and deformation on the cells. As it is obvious in Fig. 11, progress of stress on the cells starts from their nearest point to the posts. In comparison with the CTC, more parts of the leukocyte are influenced by this stress. Distribution of the stress in both cells is similar and a core-like circular pattern of stress can be determined.

Unfortunately, there is no experimental investigation on the stress of cells in DLD microfluidics comparable to our numerical one, although results presented here can be used as reliable decision-making consequences to help design DLD microfluidic devices.

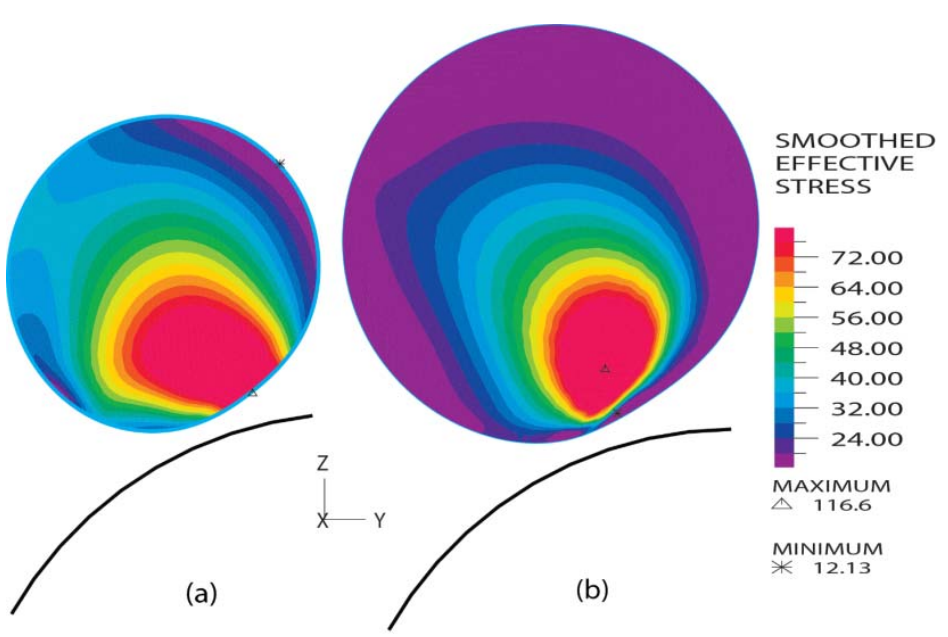

Fig. 11 A typical effective stress contour on (a) white blood cell (b) CTC and their deformations during passing beside a post. 


\section{CONCLUSIONS}

This study aimed to obtain a comprehensive analysis of a cell's motion and deformation in the DLD microfluidic device. Due to the expensive procedure of fabrication, it is vital to consider the designed microfluidic devise before fabrication; numerical analysis seems to be a powerful tool for this purpose.

Flow condition, cell deformability, and stress are crucial parameters affecting separation throughput in the DLD which all are considered in this study. In the designed DLD of this study, the CTC's motion is independent of the flow condition and is laterally displaced even for the higher Reynolds number, which is not the case for the leukocyte. By reducing the Reynolds number, e.g. decreasing the flow rate, the cell will experience a zigzag motion through arrays post. To reduce the cellular stress during separation process in order to increase the efficiency of microfluidic device [47], the obtained results of this study (Fig. 11) may be used as a significant design parameter for the DLD.

The results of this study also show the distribution of effective stress on the cell on its maximum deformation (Fig. 11). The patterns of effective stress in both CTC and leukocyte are similar; however, more areas of leukocyte experience this stress.

In conclusion, this was the first comprehensive numerical analysis of cell dynamic in the DLD microfluidic device. In this study, the interaction of the particles and flow and the cell's deformability were considered in the simulations.

\section{ACKNOWLEDGEMENTS}

The authors would like to thanks faculty members of mechanical and biomedical engineering at Amirkabir University of Technology (Tehran Polytechnic).

\section{REFERENCES}

1. Ashworth, T., "A Case of Cancer in Which Cells Similar to Those in the Tumours Were Seen in the Blood After Death,” Medical Journal of Australia, 14, pp. 146-149 (1869).

2. Norton, L. and Massague, J., "Is Cancer a Disease of Self-Seeding?," Nature Medicine, 12(8), pp. 875-878 (2006).

3. Allan, A. L. and Keeney, M., "Circulating Tumor Cell Analysis: Technical and Statistical Considerations for Application to the Clinic," Journal of Oncology (2009).

4. Williams, A., Balic, M., Datar, R. and Cote, R., "Size-Based Enrichment Technologies for CTC Detection and Characterization," Minimal Residual Disease and Circulating Tumor Cells in Breast Cancer, Springer Berlin Heidelberg, pp. 87-95 (2012).

5. Miller, M. C., Doyle, G. V. and Terstappen, L. W. M. M., "Significance of Circulating Tumor Cells Detected by the CellSearch System in Patients with
Metastatic Breast Colorectal and Prostate Cancer,” Journal of Oncology, 2010, Article ID 617421 (2009).

6. Jin, C., et al., "Technologies for Label-Free Separation of Circulating Tumor Cells: From Historical Foundations to Recent Developments," Lab on a Chip, 14, pp. 32-44 (2013).

7. Polyak, K. and Weinberg, R. A., “Transitions Between Epithelial and Mesenchymal States: Acquisition of Malignant and Stem Cell Traits," Nature Review Cancer, 9, pp. 265-273 (2009).

8. Esmaeilsabzali, H., Beischlag, T. V., Cox, M. E., Parameswaran, A. M. and Park, E. J., "Detection and Isolation of Circulating Tumor Cells: Principles and Methods,” Biotechnology Advances, 31, pp. 1063-1084 (2013).

9. Li, P., Stratton, Z. S., Dao, M., Ritz, J. and Huang, T. J., "Probing Circulating Tumor Cells in Microfluidics," Lab on a Chip, 13, pp. 602-609 (2013).

10. Movahed, S. and Li, D., “A Theoretical Study of Single-Cell Electroporation in a Microchannel," The Journal of Membrane Biology, 246, pp. 151-160 (2013).

11. Movahed, S. and Li, D., "Electrokinetic Transport of Nanoparticles to Opening of Nanopores on Cell Membrane During Electroporation," Journal of $\mathrm{Na}$ noparticle Research, 15, pp. 1-17 (2013).

12. Lim, L. S., et al., "Microsieve Lab-Chip Device for Rapid Enumeration and Fluorescence in Situ Hybridization of Circulating Tumor Cells," Lab on a Chip, 12, pp. 4388-4396 (2012).

13. Wang, G., et al., "Stiffness Dependent Separation of Cells in A Microfluidic Device," PLoS ONE, 8 (2013).

14. Warkiani, M. E., et al., "Slanted Spiral Microfluidics for the Ultra-Fast, Label-Free Isolation of Circulating Tumor Cells,” Lab on a Chip, 14, pp. 128-137 (2014).

15. Hou, H. W., et al., "Isolation and Retrieval of Circulating Tumor Cells Using Centrifugal Forces,” Scientific Reports, 3 (2013).

16. Lin, B. K., McFaul, S. M., Jin, C., Black, P. C. and Ma, H., "Highly Selective Biomechanical Separation of Cancer Cells from Leukocytes Using Microfluidic Ratchets and Hydrodynamic Concentrator," Biomicrofluidics, 7, Article ID 034114 (2013).

17. Zheng, S., et al., "3D Microfilter Device for Viable Circulating Tumor Cell (CTC) Enrichment From Blood,” Biomedical Microdevices, 13, pp. 203-213 (2011).

18. Lin, H. K., et al., "Portable Filter-Based Microdevice for Detection and Characterization of Circulating Tumor Cells," Clinical Cancer Research, 16, pp. 5011-5018 (2010).

19. Moon, H.-S., et al., "Continuous Separation of Breast Cancer Cells from Blood Samples Using Multi-Orifice Flow Fractionation (MOFF) and Dielectrophoresis (DEP)," Lab on a Chip, 11, pp. 
1118-1125 (2011).

20. Shim, S., Gascoyne, P., Noshari, J. and Hale, K. S., "Dynamic Physical Properties of Dissociated Tumor Cells Revealed by Dielectrophoretic Field-Flow Fractionation," Integrative Biology, 3, pp. 850-862 (2011).

21. Huang, L. R., Cox, E. C., Austin, R. H. and Sturm, J. C., "Continuous Particle Separation Through Deterministic Lateral Displacement,” Science, 304, pp. 987-990 (2004).

22. Zheng, S., Tai, Y. C. and Kasdan, H., “A Micro Device for Separation of Erythrocytes and Leukocytes in Human Blood," Engineering in Medicine and Biology Society, 27th Annual International Conference of the IEEE, 1, pp. 1024-1027 (2005).

23. Li, N., Kamei, D. T. and Ho, C.-M., "On-Chip Continuous Blood Cell Subtype Separation by Deterministic Lateral Displacement," 2nd IEEE International Conference on Nano/Micro Engineered and Molecular Systems, Thailand (2007).

24. Davis, J. A., et al., "Deterministic Hydrodynamics: Taking Blood Apart,” Proceedings of the National Academy of Sciences, 103, pp. 14779-14784 (2006).

25. Holm, S. H., Beech, J. P., Barrett, M. P. and Tegenfeldt, J. O., "Separation of Parasites from Human Blood Using Deterministic Lateral Displacement," Lab on a Chip, 11, pp. 1326-1332 (2011).

26. Holm, S. H., Beech, J. P., Barrett, M. P. and Tegenfeldt, J. O., “A High-Throughput Deterministic Lateral Displacement Device for Rapid and Sensitive Field-Diagnosis of Sleeping Sickness," 16th International Conference on Miniaturized Systems for Chemistry and Life Sciences. Okinawa, Japan, pp. 530-532 (2012).

27. Inglis, D. W., Herman, N. and Vesey, G., "Highly Accurate Deterministic Lateral Displacement Device and Its application to Purification of Fungal Spores," Biomicrofluidics, 4, Article ID 024109 (2010).

28. Morton, K. J., et al., "Crossing Microfluidic Streamlines to Lyse, Label and Wash Cells," Lab on a Chip, 8, pp. 1448-1453 (2008).

29. Inglis, D. W., et al., "Determining Blood Cell Size Using Microfluidic Hydrodynamics," Journal of Immunological Methods, 329, pp. 151-156 (2008).

30. Liu, Z., et al., "Rapid Isolation of Cancer Cells Using Microfluidic Deterministic Lateral Displacement Structure,” Biomicrofluidics, 7, Article ID 011801 (2013).

31. Loutherback, K., et al., "Deterministic Separation of Cancer Cells from Blood at $10 \mathrm{Ml} / \mathrm{Min}$, , AIP Advances, 2, Article ID 042107 (2012).

32. Liu, Z., et al., "High Throughput Capture of Circulating Tumor Cells Using an Integrated Microfluidic System," Biosensors and Bioelectronics, 47, pp. 113-119 (2013).

33. Long, B. R., et al., "Multidirectional Sorting Modes in Deterministic Lateral Displacement Devices," Physical Review E, 78, Article ID 046304 (2008).
34. Quek, R., Le, D. V. and Chiam, K. H., "Separation of Deformable Particles in Deterministic Lateral Displacement Devices," Physical Review E, 83, Article ID 056301 (2011).

35. D’Avino, G., "Non-Newtonian Deterministic Lateral Displacement Separator: Theory and Simulations," Rheologica Acta, 52, pp. 221-236 (2013).

36. Inglis, D. W., Davis, J. A., Austin, R. H. and Sturm, J. C., "Critical Particle Size for Fractionation by Deterministic Lateral Displacement," Lab on a Chip, 6, pp. 655-658 (2006).

37. Meng, S., et al., "Circulating Tumor Cells in Patients with Breast Cancer Dormancy,” Clinical Cancer Research, 10, pp. 8152-8162 (2004).

38. Beech, J. P., Holm, S. H., Adolfsson, K. and Tegenfeldt, J. O., "Sorting Cells by Size, Shape and Deformability," Lab on a Chip, 12, pp. 1048-1051 (2012).

39. Inglis, D. W., et al., "Microfluidic Device for Label-Free Measurement of Platelet Activation," Lab on a Chip, 8, pp. 925-931 (2008).

40. Chen, C. L., et al., "Single- Cell Analysis of Circulating Tumor Cells Identifies Cumulative Expression Patterns of EMT-Related Genes in Metastatic Prostate Cancer,” Prostate, 73, pp. 813-826 (2013).

41. Shirai, A. and Masuda, S., "Numerical Simulation of Passage of a Neutrophil Through a Rectangular Channel with a Moderate Constriction," PLoS One, 8, Article ID e59416 (2013).

42. Bathe, M., Shirai, A., Doerschuk, C. M. and Kamm, R. D., "Neutrophil Transit Times Through Pulmonary Capillaries: The Effects of Capillary Geometry and Fmlp-Stimulation,” Biophysical Journal, 83, pp. 1917-1933 (2002).

43. Donea, J., Giuliani, S. and Halleux, J. P., “An Arbitrary Lagrangian-Eulerian Finite Element Method for Transient Dynamic Fluid-Structure Interactions,” Computer Methods in Applied Mechanics and Engineering, 33, pp. 689-723 (1982).

44. Bathe, K.-J. and Zhang, H., "A Mesh Adaptivity Procedure for CFD and Fluid-Structure Interactions," Computers \& Structures, 87, pp. 604-617 (2009).

45. Vahidi, B. and Fatouraee, N., "Large Deforming Buoyant Embolus Passing Through a Stenotic Common Carotid Artery: A Computational Simulation,” Journal of Biomechanics, 45, pp. 1312-1322 (2012).

46. Kulrattanarak, T., Sman, R. G. M., Schroën, C. G. P. H. and Boom, R. M., "Analysis of Mixed Motion in Deterministic Ratchets Via Experiment and Particle Simulation," Microfluidics and Nanofluidics, 10, pp. 843-853 (2011).

47. Kuo, J. S., et al., "Deformability Considerations in Filtration of Biological Cells," Lab on a Chip, 10, pp. 837-842 (2010).

(Manuscript received May 15, 2015, accepted for publication July 12, 2015.) 